\title{
Effects of Ag Nanocubes with Different Corner Shape on the Absorption Enhancement in Organic Solar Cells
}

\author{
Feng Shan, ${ }^{1,2}$ Tong Zhang, ${ }^{1,2}$ and Sheng-Qing $\mathrm{Zhu}^{1,2}$ \\ ${ }^{1}$ School of Electronic Science and Engineering, Key Laboratory of Micro-Inertial Instrument and Advanced Navigation Technology, \\ Ministry of Education, Southeast University, Nanjing, Jiangsu 210096, China \\ ${ }^{2}$ Suzhou Key Laboratory of Metal Nano-Optoelectronic Technology, Suzhou Research Institute of Southeast University, Suzhou, \\ Jiangsu 215123, China
}

Correspondence should be addressed to Tong Zhang; tzhang@seu.edu.cn

Received 28 February 2014; Revised 24 April 2014; Accepted 24 April 2014; Published 19 May 2014

Academic Editor: Jing Chen

Copyright (C) 2014 Feng Shan et al. This is an open access article distributed under the Creative Commons Attribution License, which permits unrestricted use, distribution, and reproduction in any medium, provided the original work is properly cited.

\begin{abstract}
The effects of corner shape of silver (Ag) nanocubes (NCs) on optical absorptions of organic solar cells (OSCs) are theoretically investigated by finite element method (FEM) calculations. The absorption of sun light in the active layer is calculated. Significant absorption enhancements have been demonstrated in metallic region with different shapes of $\mathrm{Ag} \mathrm{NCs}$, among them corner radius (R) is zero result in the best light absorption performance of up to 55\% enhancement with respect to bare OSCs. The origins of increased absorption are believed to be the effects of the huge electric field enhancement and increased scattering upon the excitation of localized surface plasmon resonance (LSPR). Apart from using $R=0$, we show that $R=3,6$, and 11.29 of Ag NCs in metallic region of active layer may also result in the maximum comparable absorption enhancement of $49 \%, 41 \%$, and $28 \%$, respectively. In addition, a significant effect of the period of NCs is observed.
\end{abstract}

\section{Introduction}

Organic solar cells (OSCs) have attracted considerable attentions due to that they are lightweight, flexible, inexpensive to fabricate, and tunable on the molecular level $[1,2]$. The active layers of most OSCs, however, are not thicker than $200 \mathrm{~nm}$, which is not thick enough to harvest all the solar radiation below their optical band gaps [3]. This limitation of the thickness of the active layers is mostly due to short charge carrier diffusion lengths stemming from their poor charge carrier mobility. The advanced bulk heterojunction (BHJ) concept was introduced to solve the diffusion length problem and to keep the required thickness of the active layer for sufficient light absorption $[4,5]$. However, even with a BHJ, the thickness cannot be too large. When the thickness is above a certain value, the conversion efficiency will drop since free carrier recombination becomes significant [6]. To overcome this problem, several approaches including the utilization of low-band gap polymers that absorb the red and infrared parts of the solar spectrum, the application of periodic nanostructure for light trapping in the active layer and metallic electrode, and the incorporation of metallic nanostructures to increase the absorption of organic materials due to the high electromagnetic field strength in the vicinity of the excited surface plasmons have been reported [7-14].

Several works have demonstrated that the use of metallic nanostructures in solar cells results in optical field enhancement and improvement of the optical absorption [15-19]. The metallic nanostructure not only can scatter and couple the incident light into the solar cell [16, 20-24] but also confine the light surrounding their surfaces [19,25-27], which results in the light absorption enhancement of solar OCSs. These plasmonic light trapping systems offer two main mechanisms to concentrate light, localized surface plasmon resonance (LSPR) and surface plasmon polaritons (SPP) [28]. The LSPR is a characteristic of small metallic nanoparticles and yields very high field enhancement within a few nanometers from the metallic surface. Moreover, it was theoretically demonstrated that embedding metallic nanoparticles in active layers leads to much greater absorption enhancements compared with the case with metallic nanoparticles in buffer layers [29, 


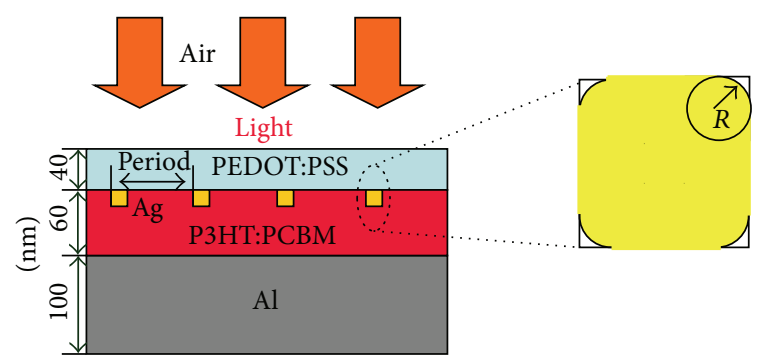

FIGURE 1: Schematic of periodic Ag NCs embedded in the active layer of OSCs.

30]. Moreover, the enhancement effects of gold nanoparticles on the photocurrent and energy conversion efficiency have been observed in experiments [30].

In this paper, we theoretically investigated the effect of introducing metallic nanocubes (NCs) with different shape in OSCs on their optical absorption enhancement. More specifically, we considered OSCs in which the active layer is partially substituted by periodic silver (Ag) NCs. We found that metallic NCs led to great absorption enhancement in $60 \mathrm{~nm}$ thick blended polymer, P3HT: PCBM (poly-3-hexythiphene and phenyl-C61-butyric acid methyl ester), which is one of the most widely used materials for polymer solar cells. This is due to the large field enhancement of the metallic NCs, associated with the LSPR. Here, a concrete OSCs structure based on the finite element method (FEM) was modeled. Actually, LSPR mode in $3 \mathrm{D}$ nanostructures composed of periodic Au or Ag nanoparticles both can be excited by TE and TM polarized light, and they have the equivalent effect for centrosymmetric nanoparticles. However, the calculation in $3 \mathrm{D}$ simulation would take a lot of time and computer memory. Therefore we reduced the $3 \mathrm{D}$ model to a $2 \mathrm{D}$ one, since previous researchers have shown that a $2 \mathrm{D}$ model can give similar results to a $3 \mathrm{D}$ model [31]. In 2D case, plasmonic excitation is possible only under TM illumination.

\section{Simulation Model and Methods}

A 2D cross-section image of OSCs in this study is illustrated in Figure 1. The hole transport layer is made of $40 \mathrm{~nm}$ thick PEDOT: PSS, poly(3, 4-ethylenedioxythiophene): poly(styrenesulfonate), which is a polymer with good chemical and thermal stability and good flexibility. The commonly used active layer BHJ material consists of the electron donor P3HT and the electron acceptor PCBM. Results for $60 \mathrm{~nm}$ thick polymer layer P3HT : PCBM with 1:1 weight ratio are presented here, in contact with the cathode made of aluminum ( $\mathrm{Al}$ ) with $100 \mathrm{~nm}$ thick. The material of the metallic nanoparticles is Ag NCs. The material properties of PEDOT: PSS, P3HT : PCBM, Al, and Ag are taken from literature $[32,33]$.

The NCs are embedded into the top of active layer in the form of periodic array. As shown in Figure 1, we assume the light as incident plane wave illuminates on the OSCs. The incident plane wave is the TM polarization under wavelengths of $300-800 \mathrm{~nm}$ which is the region of interest for

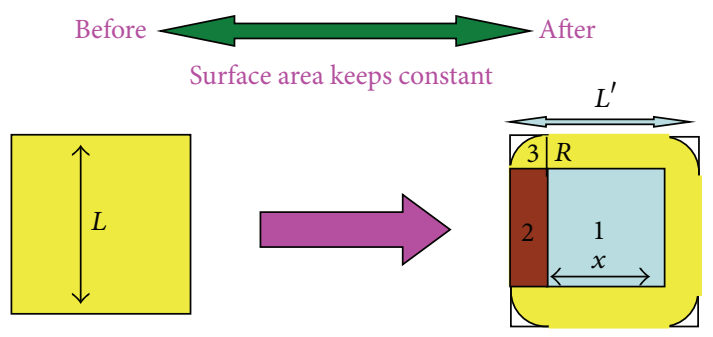

FIGURE 2: Surface area of Ag NCs keeps constant before and after $R$ changes.

TABLE 1: (Unit: $\mathrm{nm}) L=20$.

\begin{tabular}{llll}
\hline$R=0$ & $R=3$ & $R=6$ & $R=11.29$ \\
$L^{\prime}=20$ & $L^{\prime}=20.19$ & $L^{\prime}=20.76$ & $L^{\prime}=22.58$ \\
\hline
\end{tabular}

the active layer material. The light illuminates from the air into the OSCs passing the whole transport layer and the active layer and reaching the cathode. In the FEM model, periodic boundary conditions are used at the left and right boundaries, while perfectly matched layer (PML) absorbing boundary conditions are used at the top and bottom boundaries of the simulation domain. The absorption in the active layer is calculated by integrating the divergence of the Poynting vector (power flow) which is then normalized with input power.

As well known, metallic NCs dispersed in the active layer of OSCs can enhance the absorption due to the LSPR of NCs. However, the scattering and absorption of NCs are highly size- and shape-dependent $[34,35]$. We modeled the layouts both with and without the metallic NCs. In the optimization of the cell geometry we simultaneously varied the shape and the period of Ag NCs, as shown in Figure 2. In order to ensure comparability among OSCs with different shape, the occupation ratio of $\mathrm{Ag} \mathrm{NCs}$ in active material must be consistent; therefore we kept the surface area as a constant. As can be seen from Figure 2, by increasing the corner radius from zero to $R$, the side lengths altered from $L$ to $L^{\prime}$. In view of geometry relation of surface area before and after changes, $L^{\prime}$ can be calculated at different $R$

$$
\begin{gathered}
L^{2}=x^{2}+4 x R+\pi R^{2} \\
L^{\prime}=x+2 R,
\end{gathered}
$$

where $R$ is the corner radius of Ag NCs and $x$ is the side lengths of region 1 in Figure 2. The surface area of Ag NCs is fixed at $400 \mathrm{~nm}^{2}$, and then $L=20 \mathrm{~nm}(R=0 \mathrm{~nm})$. We can get the value $L^{\prime}$ from (1) corresponding to $R=3,6$, and 11.29 as shown in Table 1.

\section{Results and Discussions}

We investigated the influence of the $R$ on the absorption. In order to reveal the mechanism of absorption enhancement, the side lengths $L^{\prime}$ of the metallic NCs were fixed in Table 1. To quantify the absorption enhancement within the polymer itself, we integrated the Poynting vector $\vec{S}$ within the region 


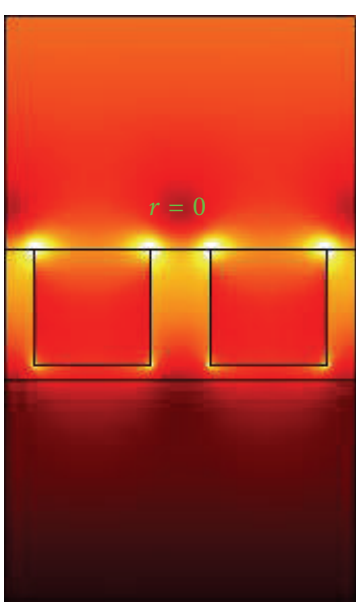

(a)

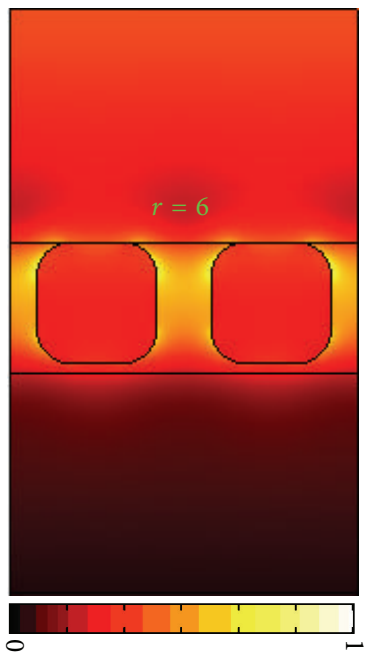

(c)

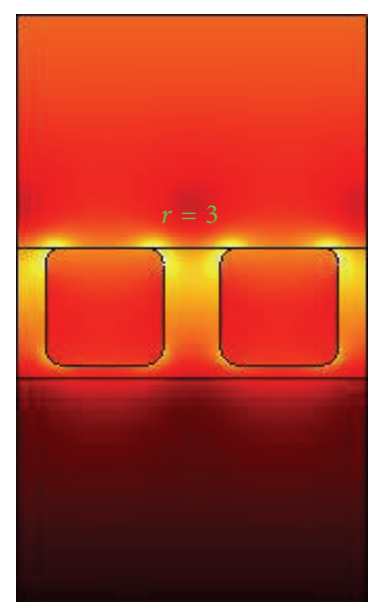

(b)

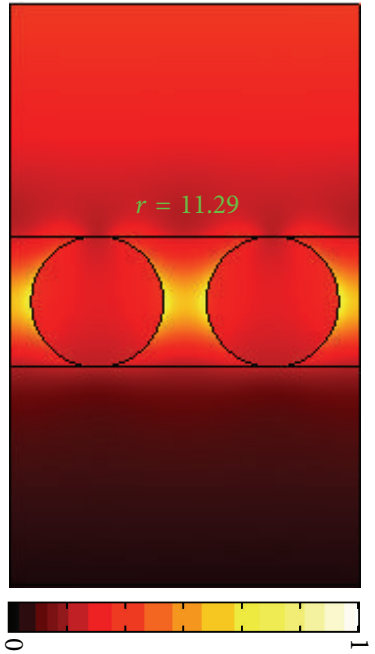

(d)

FIGURE 3: Electric field intensity for incident light at a wavelength of $480 \mathrm{~nm}$, the period of $30 \mathrm{~nm}$ and $R$ of Ag NCs are (a) 0, (b) 3, (c) 6, and (d) $11.29 \mathrm{~nm} . \lambda=480 \mathrm{~nm}, P=30 \mathrm{~nm}$.

only and normalized it to the incoming power over one period $P_{\text {inc }}$

$$
A(\lambda)=\frac{\int(\nabla \cdot S) d V_{\text {polymer }}}{P_{\text {inc }}}
$$

To quantity the absorption capability of the solar cell structure in the wavelength region of interest, we defined a normalized weighted integrated absorption for the AM $1.5 \mathrm{G}$ spectrum

$$
A_{\text {int }}=\frac{\int\left[P_{A 1.5 \mathrm{G}}(\lambda) \times A(\lambda)\right] d \lambda}{\int P_{\mathrm{AM} 1.5 \mathrm{G}}(\lambda) d \lambda} .
$$

Our choice of the $300-800 \mathrm{~nm}$ wavelength regions is relative to the absorption properties of active layer material. We can obtain the absorption enhancement (ratio of absorption with metallic NCs to that without metallic NCs) in the active layer (it does not include the absorption in the metallic NCs) in function of metallic NCs period

$$
\text { Absorption Enhancement }=\frac{A_{\text {int }}(\text { with NPs })}{A_{\text {int }}(\text { without NPs })},
$$

where $A_{\text {int }}$ (with NCs) and $A_{\text {int }}$ (without NCs) are the normalized weighted integrated absorption with and without metallic NCs in the active layer, respectively.

Exact analytical solutions for metallic NCs interacting with electromagnetic radiation have been investigated by Mie in the stationary case for metallic spheres [36]. Here we consider NCs, but some general principles proven for spheres are still valid. The plasmonic effects can be attributed to the coherent oscillations of free electrons within the metallic NCs. By comparing the resonance wavelengths, we found that small NCs acted as a forced dipole oscillator with a resonance wavelength set by the dielectric permittivity of the surrounding medium and the dispersive permittivity of the metal. In general, the resonance wavelength will depend on the shape of the NCs and its size. 


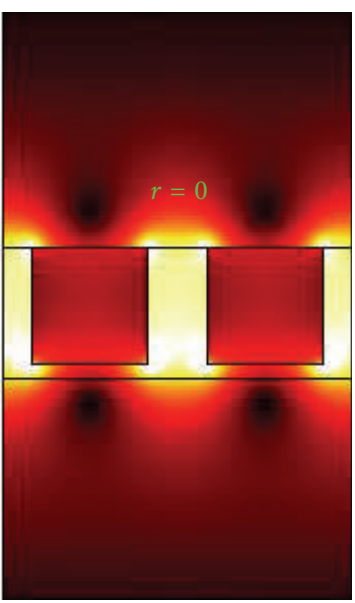

(a)

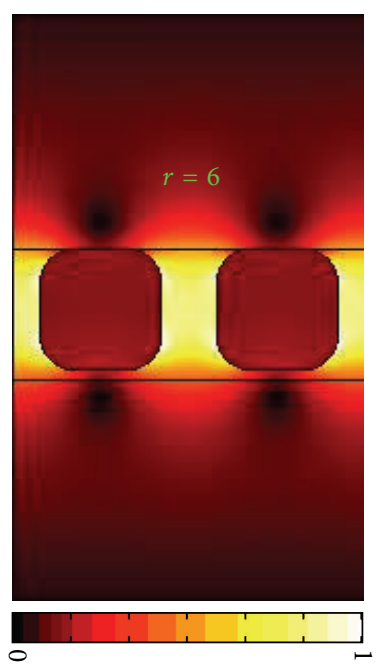

(c)

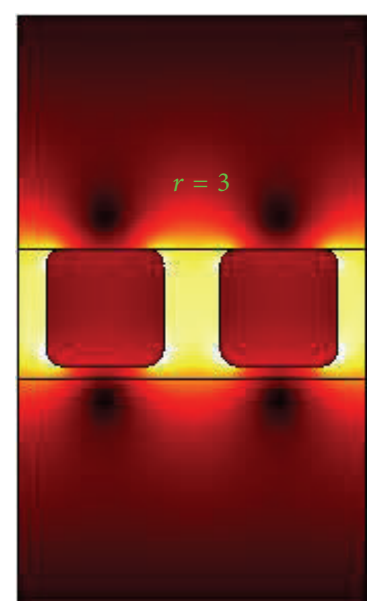

(b)

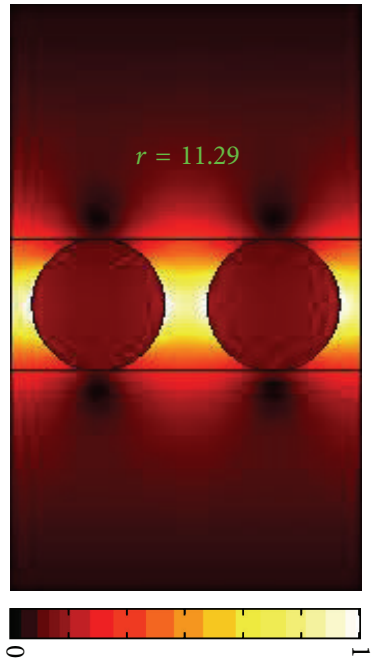

(d)

FIGURE 4: Electric field intensity for incident light at a wavelength of $750 \mathrm{~nm}$, the period of $30 \mathrm{~nm}$ and $R$ of Ag NCs are (a) 0, (b) 3, (c) 6, and (d) $11.29 \mathrm{~nm}$. $\lambda=750 \mathrm{~nm}, P=30 \mathrm{~nm}$.

Figure 3 shows the calculated electric field intensity in the $x-y$ plane, at a wavelength of $480 \mathrm{~nm}$. The Ag NCs have a period of $30 \mathrm{~nm}$ and the $R$ of (a) 0 , (b) 3, (c) 6 , and (d) $11.29 \mathrm{~nm}$. It is noted that the electric field is distributed around the particle. The localized surface plasmon modes excited at the interface between the Ag NCs and the active layer cause strong field enhancement around the interface. The field is mainly situated in the active layer rather than inside the metal, which brings in an improvement in the absorption efficiency of the active layer, particularly around the edges of the Ag NCs. The amplified electric field intensity penetrates into the active layers, leading to absorption enhancements. With varying the $R$ of the Ag NCs, the electric fields distributed were calculated and plotted in Figure 3. A very high value of the electric field can be observed on the NCs surface, and as can be seen from Figure 3(a), the electric field distribution at the corner of nanocubes is more concentrated than other regions. As the $R$ increased, the charge density on the NCs decreases leading to the decrease of the absolute value of the surface electric field on the NCs, as can be observed in Figures 3(a), 3(b), 3(c), and 3(d).

One might argue that only giving the electric field intensity distribution in the active layer at a monochromatic wave is not adequate to demonstrate the enhancement mechanisms; to this end, we examined the electric field intensity distribution at a wavelength of $750 \mathrm{~nm}$ and the period of $30 \mathrm{~nm}$ (Figure 4). Interestingly, Figure 4 shows a larger electric field intensity enhancement in the wavelength of $750 \mathrm{~nm}$ than the wavelength of $480 \mathrm{~nm}$, owing to that geometry of NCs and the incidence wavelengths are more close to the resonance condition.

We theoretically investigated the influence of the corner shape of Ag NCs on the optical absorption in thin OSCs. In order to reveal the enhancement mechanism of near field and scattering, the active layer was divided into three sublayers: parts I, II, and III sublayers, as depicted in the inset of Figure 5(a), Figure 6(a), and Figure 7(a). The complex refractive index of Ag was modeled by Drude-Lorentz model. 


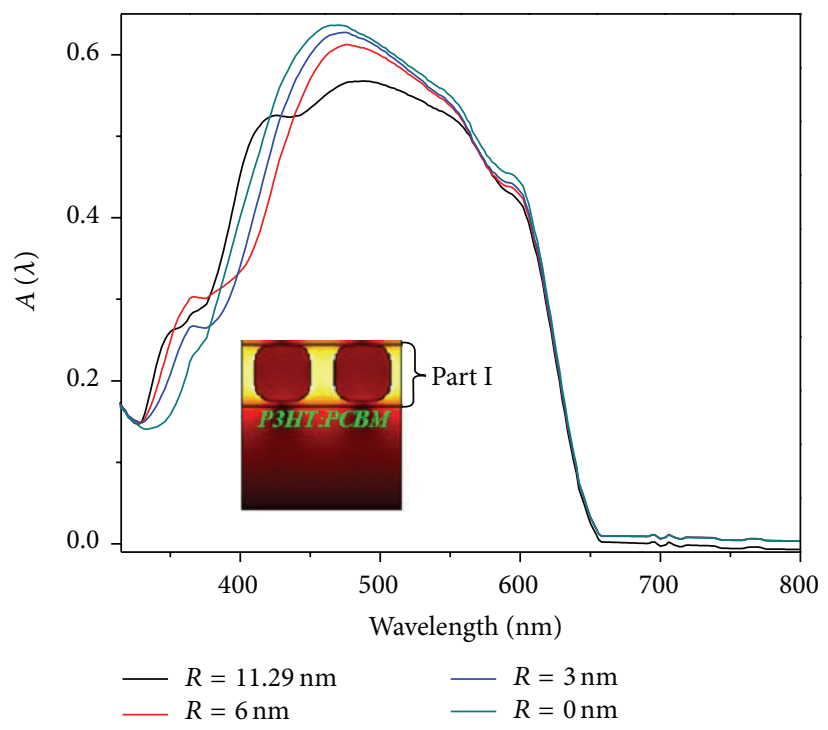

(a)

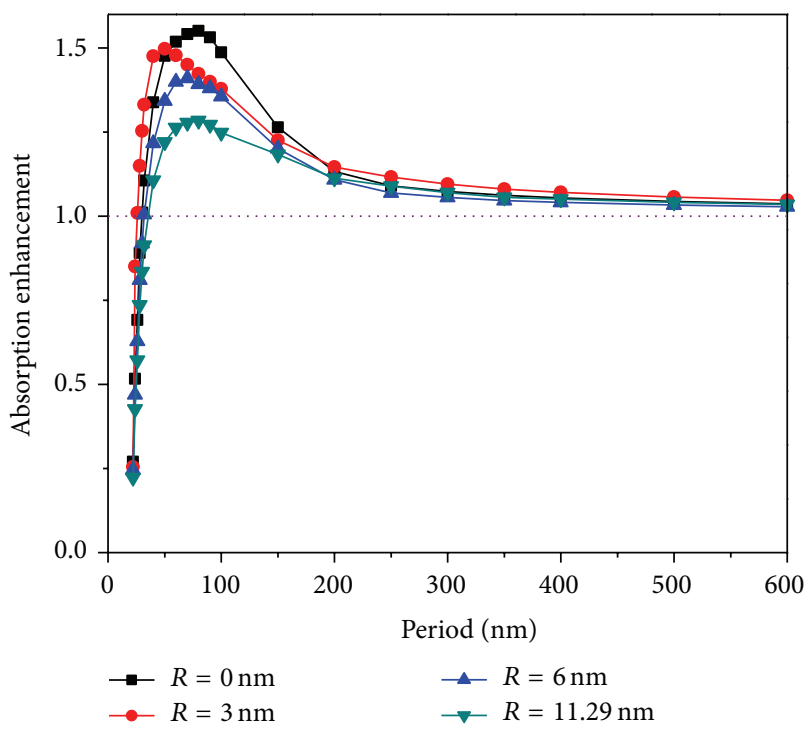

(b)

Figure 5: (a) Absorption spectra in the active layer of part I of the OSCs with Ag NCs of different $R$. (b) Absorption enhancement in the active layer of part I exhibited by OSCs relative to a pristine solar cell.

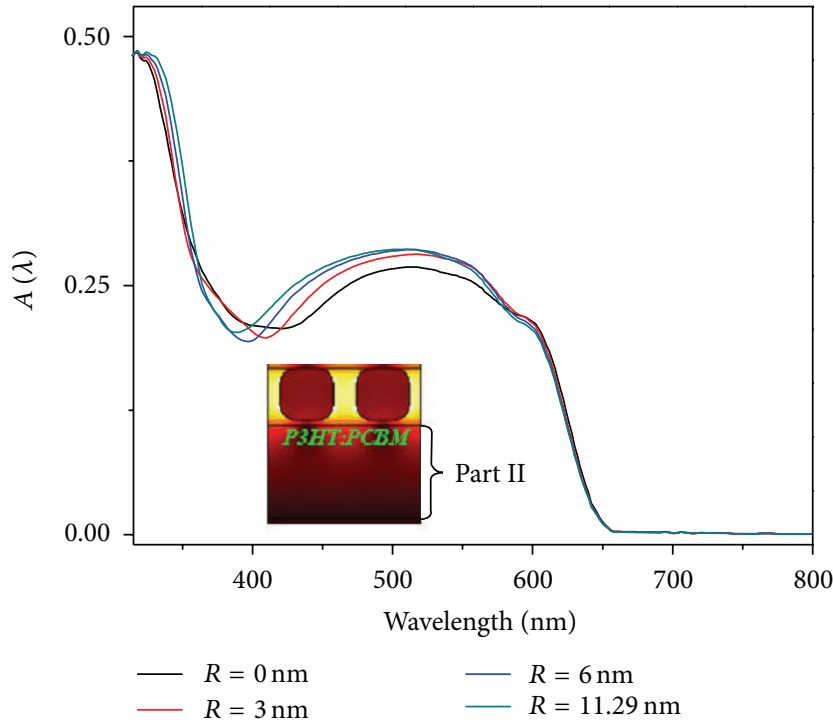

(a)

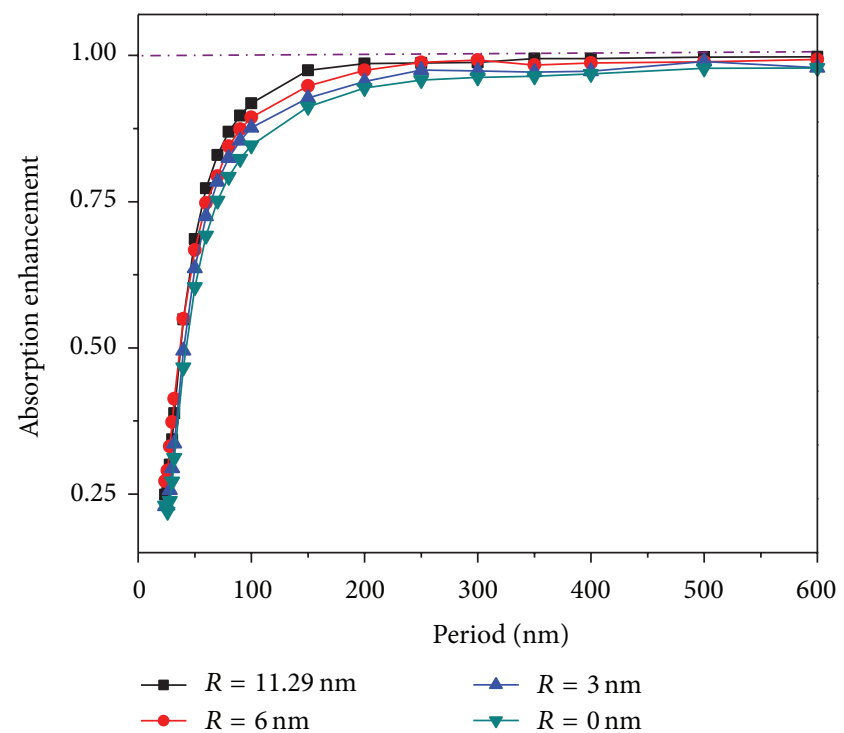

(b)

Figure 6: (a) Absorption spectra in the active layer of part II of the OSCs with Ag NCs of different $R$. (b) Absorption enhancement in the active layer of part II exhibited by OSCs relative to a pristine solar cell.

Figure 5(a) shows the calculated absorption spectrum in the active layer of part I, and Ag NCs with different corner shape integrated inside the active layer. It was noted that the absorption is obviously decreased with the $R$ increased at short wavelength, which was consistent with the electric field distributed in Figure 4 . The absorption enhancement in the active layer of part I is shown in Figure 5(b) for varying $R$ of $\mathrm{Ag} \mathrm{NCs}$ and periods ranging up to $600 \mathrm{~nm}$. It is important to note that the volume of active material in this configuration is decreased due to the presence of the Ag NCs. However, the total amount of light absorbed in the active layer of part I increased. The absorption enhancement due to the presence of the Ag NCs outweighs the reduction in absorption due to the omitted active material. The enhancement is owed to the excitation of localized surface plasmon modes at the interface between the Ag NCs and the active layer. A maximum absorption enhancement of $55 \%$ is observed at the period of $80 \mathrm{~nm}$ and $R=0$. At small periods the content of active material rapidly decreases and the active absorption drops correspondingly. At large periods, where the density of 


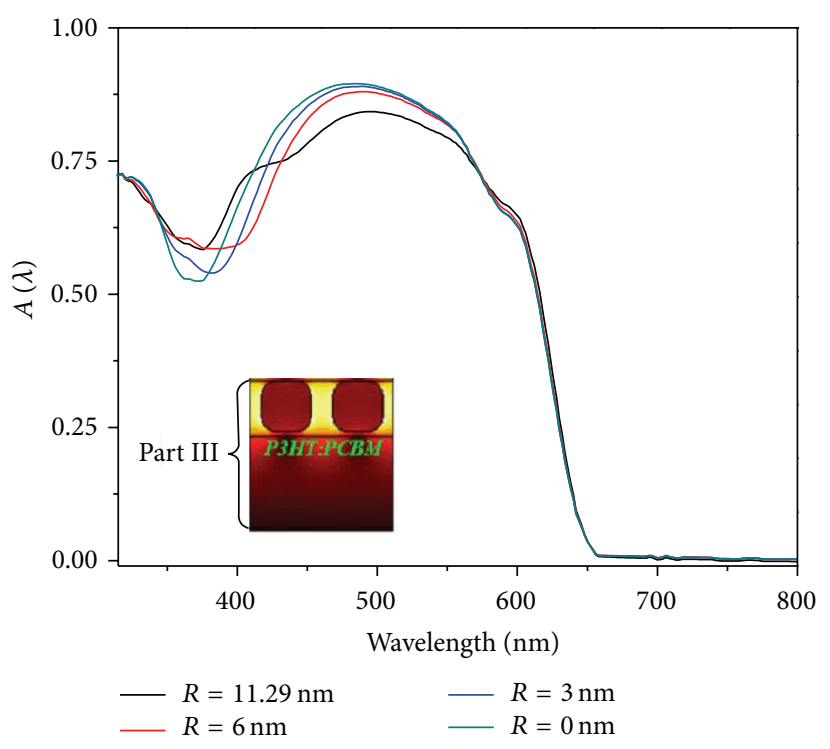

(a)

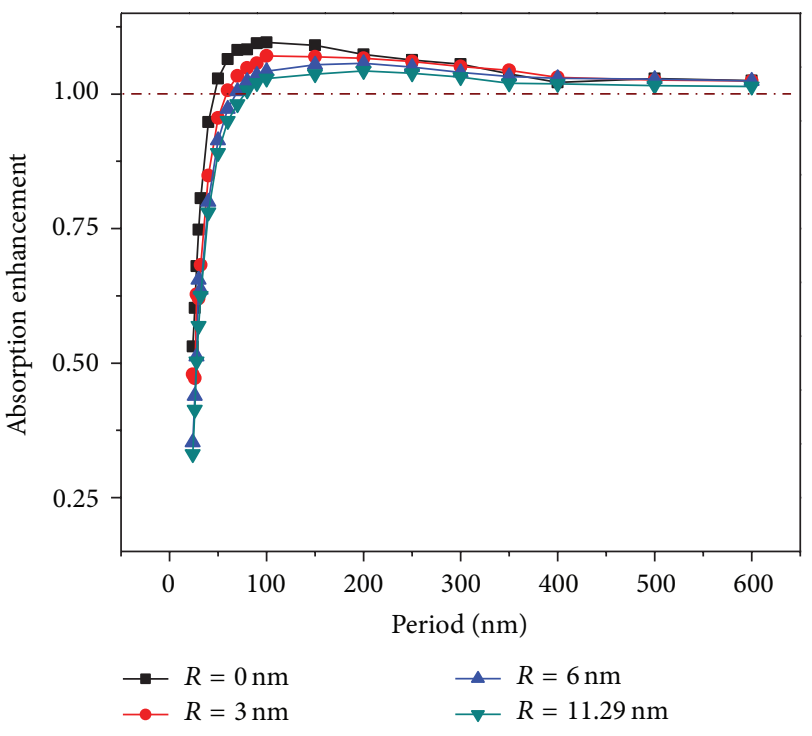

(b)

FIgure 7: (a) Absorption spectra in the active layer of part III of the OSCs with Ag NCs of different R. (b) Absorption enhancement in the active layer of part III exhibited by OSCs relative to a pristine solar cell.

features becomes small, the absorption enhancement tends towards the value of 1 , as expected. The peak of the curve indicates that light trapping ability is the strongest. We can see from Figure 5(b) that the absorption enhancement is decreased with the increase of $R$, which is corresponding to the electric distribution as shown in Figure 4. The LSPR frequency strongly depends on the shape of nanostructure. For the periodic NCs array, it is identified that the absorption resonance is mainly due to gap plasmon when the separation distance between neighboring NCs is small enough. When the distance becomes larger, the field strength in the gap region weakens sharply, and the resonance is dominated by the shape of NC. Therefore at a distance value (i.e., 200 $400 \mathrm{~nm}$ ), the resonance can be an equal mixture of the two above mentioned resonances. For the nanostructures with different shapes, the separation distance of the appearance of gap plasmon is different. So it is an interesting phenomenon that the NCs with $11.29 \mathrm{~nm}$ corner radius show higher enhancement than those with $6 \mathrm{~nm}$ one from $200 \mathrm{~nm}$ to $400 \mathrm{~nm}$ period (the distance between the neighbor NCs) in Figure 5(b). This can be ascribed to the fact that the distances of interparticle gap for NCs with corner radius of $11.29 \mathrm{~nm}$ and $6 \mathrm{~nm}$ are different.

Figure 6(a) shows the absorption spectrum in part II of the active layer with a periodic array of Ag NCs, at various $R$. As can be seen from Figure 6(a), absorption intensity increases with the increase of $R$. Because the incoming power into OSCs is a constant, part I has a high field enhancement due to the LSPR performance of nanocubes, leading to a reduced power entry into part II. The absorption enhancement can be observed in Figure 6(b). The absorption enhancement ratio increases sharply at first and then decays steadily with the period increases. In addition, the absorption increases with the increase of $R$. Figures 7(a) and 7(b) show the absorption spectra and the absorption enhancement for $\mathrm{Ag}$ NCs of various $R$ in part III of the active layer. It can be seen that the absorption enhancement with Ag NCs has a significant increase compared with that without Ag NCs, which may result from the LSPR enhanced electric field. The absorption enhancements among different corner shapes of NCs are different. When the corner radius is zero, the enhancement is the highest.

\section{Conclusion}

We investigated the absorption enhancement induced by Ag NCs with different $R$ within active layers, and then the influence of $R$ on the absorption was analyzed. We found that the LSPR modes excited by the Ag NCs led to strong absorption enhancement. Significant absorption enhancements had been demonstrated in metallic region with various $R$ of $\mathrm{Ag}$ NCs, among them $R=0$ resulted in the best light absorption performance of up to $55 \%$ enhancement with respect to bare OSCs. The origins of increased absorption are believed to be the effects of the huge electric field enhancement and increased scattering upon the excitation of localized surface plasmon resonance. Apart from using $R=0$, we also showed that $R=3,6$, and $11.29 \mathrm{~nm}$ of Ag NCs in metallic region of active layer may result in the maximum comparable absorption enhancement of $49 \%, 41 \%$, and $28 \%$, respectively. Furthermore, this work also provided better understanding of the enhancement mechanism by Ag NCs in photovoltaic devices.

\section{Conflict of Interests}

The authors declare that there is no conflict of interests regarding the publication of this paper. 


\section{Acknowledgments}

This work is supported by NSFC under Grant no. 61307066, Doctoral Fund of Ministry of Education of China under Grant nos. 20110092110016 and 20130092120024, Natural Science Foundation of Jiangsu Province under Grant no. BK20130630, the National Basic Research Program of China (973 Program) under Grant no. 2011CB302004, and the Foundation of Key Laboratory of Micro-Inertial Instrument and Advanced Navigation Technology, Ministry of Education, China, under Grant no. 201204.

\section{References}

[1] G. Li, V. Shrotriya, J. Huang et al., "High-efficiency solution processable polymer photovoltaic cells by self-organization of polymer blends," Nature Materials, vol. 4, no. 11, pp. 864-868, 2005.

[2] C. Deibel and V. Dyakonov, "Polymer-fullerene bulk heterojunction solar cells," Reports on Progress in Physics, vol. 73, no. 9, Article ID 096401, 2010.

[3] I. Kim, D. S. Jeong, T. S. Lee, W. S. Lee, and K. S. Lee, "Plasmonic nanograting design for inverted polymer solar cells," Optics Express, vol. 20, no. 105, pp. A729-A739, 2012.

[4] T. Soga, Nanostructured Materials for Solar Energy Conversion, Elsevier, Amsterdam, The Netherlands, 2006.

[5] G. Yu, J. Gao, J. C. Hummelen, F. Wudl, and A. J. Heeger, "Polymer photovoltaic cells: enhanced efficiencies via a network of internal donor-acceptor heterojunctions," Science, vol. 270, no. 5243, pp. 1789-1791, 1995.

[6] M. Gu, Z. Ouyang, B. Jia et al., "Nanoplasmonics: a frontier of photovoltaic solar cells," Nanophotonics, vol. 1, no. 3-4, pp. 235248, 2012.

[7] C. Winder and N. S. Sariciftci, "Low bandgap polymers for photon harvesting in bulk heterojunction solar cells," Journal of Materials Chemistry, vol. 14, no. 7, pp. 1077-1086, 2004.

[8] A. J. Morfa, K. L. Rowlen, T. H. Reilly III, M. J. Romero, and J. van de Lagemaat, "Plasmon-enhanced solar energy conversion in organic bulk heterojunction photovoltaics," Applied Physics Letters, vol. 92, no. 1, Article ID 013504, 2008.

[9] J. Y. Kim, S. H. Kim, H.-H. Lee et al., "New architecture for high-efficiency polymer photovoltaic cells using solution-based titanium oxide as an optical spacer," Advanced Materials, vol. 18, no. 5, pp. 572-576, 2006.

[10] C. Cocoyer, L. Rocha, L. Sicot et al., "Implementation of submicrometric periodic surface structures toward improvement of organic-solar-cell performances," Applied Physics Letters, vol. 88, no. 13, Article ID 133108, 2006.

[11] S.-I. Na, S.-S. Kim, S.-S. Kwon et al., "Surface relief gratings on poly(3-hexylthiophene) and fullerene blends for efficient organic solar cells," Applied Physics Letters, vol. 91, no. 17, Article ID 173509, 2007.

[12] M. C. Scharber, D. Mühlbacher, M. Koppe et al., "Design rules for donors in bulk-heterojunction solar cells-towards $10 \%$ energy-conversion efficiency," Advanced Materials, vol. 18, no. 6, pp. 789-794, 2006.

[13] X. Wang, E. Perzon, F. Oswald et al., "Enhanced photocurrent spectral response in low-bandgap polyfluorene and $\mathrm{C}_{70}{ }^{-}$ derivative-based solar cells," Advanced Functional Materials, vol. 15, no. 10, pp. 1665-1670, 2005.
[14] M. N. Lin, S. Q. Zhu, X. Y. Zhang, T. Zhang, and D. Su, "Island shaped gold nanoparticle film with controlable optical properties for surface enhanced Raman scattering," submitted to NRL Advances.

[15] N. C. Lindquist, W. A. Luhman, S.-H. Oh, and R. J. Holmes, "Plasmonic nanocavity arrays for enhanced efficiency in organic photovoltaic cells," Applied Physics Letters, vol. 93, no. 12, Article ID 123308, 2008.

[16] N. C. Panoiu and R. M. Osgood Jr., "Enhanced optical absorption for photovoltaics via excitation of waveguide and plasmonpolariton modes," Optics Letters, vol. 32, no. 19, pp. 2825-2827, 2007.

[17] K. R. Catchpole and A. Polman, "Plasmonic solar cells," Optics Express, vol. 16, no. 26, pp. 21793-21800, 2008.

[18] R. A. Pala, J. White, E. Barnard, J. Liu, and M. L. Brongersma, "Design of plasmonic thin-film solar cells with broadband absorption enhancements," Advanced Materials, vol. 21, no. 34, pp. 3504-3509, 2009.

[19] H. Shen, P. Bienstman, and B. Maes, "Plasmonic absorption enhancement in organic solar cells with thin active layers," Journal of Applied Physics, vol. 106, no. 7, Article ID 073109, 2009.

[20] D. Qu, F. Liu, J. Yu et al., "Plasmonic core-shell gold nanoparticle enhanced optical absorption in photovoltaic devices," Applied Physics Letters, vol. 98, no. 11, Article ID 113119, 2011.

[21] S. Pillai, K. R. Catchpole, T. Trupke, and M. A. Green, "Surface plasmon enhanced silicon solar cells," Journal of Applied Physics, vol. 101, no. 9, Article ID 093105, 2007.

[22] S. H. Lim, W. Mar, P. Matheu, D. Derkacs, and E. T. Yu, "Photocurrent spectroscopy of optical absorption enhancement in silicon photodiodes via scattering from surface plasmon polaritons in gold nanoparticles," Journal of Applied Physics, vol. 101, no. 10, Article ID 104309, 2007.

[23] D. Derkacs, S. H. Lim, P. Matheu, W. Mar, and E. T. Yu, "Improved performance of amorphous silicon solar cells via scattering from surface plasmon polaritons in nearby metallic nanoparticles," Applied Physics Letters, vol. 89, no. 9, Article ID 093103, 2006.

[24] L. Yang, Y. Xuan, and J. Tan, "Efficient optical absorption in thin-film solar cells," Optics Express, vol. 19, no. 19, pp. A1165A1174, 2011.

[25] B. P. Rand, P. Peumans, and S. R. Forrest, "Long-range absorption enhancement in organic tandem thin-film solar cells containing silver nanoclusters," Journal of Applied Physics, vol. 96, no. 12, pp. 7519-7526, 2004.

[26] M. Xue, L. Li, B. J. T. de Villers et al., "Charge-carrier dynamics in hybrid plasmonic organic solar cells with Ag nanoparticles," Applied Physics Letters, vol. 98, no. 25, Article ID 253302, 2011.

[27] D. Qu, F. Liu, X. Pan et al., "Plasmonic core-shell nanoparticle enhanced optical absorption in thin film organic solar cells," in Proceedings of the 37th IEEE Photovoltaic Specialists Conference (PVSC '11), pp. 000924-000928, IEEE, 2011.

[28] S. A. Maier, Plasmonics: Fundamentals and Applications: Fundamentals and Applications, Springer, New York, NY, USA, 2007.

[29] W. E. I. Sha, W. C. H. Choy, Y. G. Liu, and W. C. Chew, "Near-field multiple scattering effects of plasmonic nanospheres embedded into thin-film organic solar cells," Applied Physics Letters, vol. 99, no. 11, Article ID 113304, 2011.

[30] D. H. Wang, D. Y. Kim, K. W. Choi et al., "Enhancement of donor-acceptor polymer bulk heterojunction solar cell power conversion efficiencies by addition of Au nanoparticles," Angewandte Chemie -International Edition, vol. 50, no. 24, pp. 5519-5523, 2011. 
[31] F. Moreno, B. García-Cámara, J. M. Saiz, and F. González, "Interaction of nanoparticles with substrates: effects on the dipolar behaviour of the particles," Optics Express, vol. 16, no. 17, pp. 12487-12504, 2008.

[32] E. D. Palik, Handbook of Optical Constants of Solids, Academic Press, New York, NY, USA, 1998.

[33] F. Monestier, J.-J. Simon, P. Torchio et al., "Modeling the short-circuit current density of polymer solar cells based on P3HT:PCBM blend," Solar Energy Materials and Solar Cells, vol. 91, no. 5, pp. 405-410, 2007.

[34] J.-Y. Lee and P. Peumans, "The origin of enhanced optical absorption in solar cells with metal nanoparticles embedded in the active layer," Optics Express, vol. 18, no. 10, pp. 10078-10087, 2010.

[35] S. Pillai and M. A. Green, "Plasmonics for photovoltaic applications," Solar Energy Materials and Solar Cells, vol. 94, no. 9, pp. 1481-1486, 2010.

[36] G. Mie, "Beiträge zur Optik trüber Medien, speziell kolloidaler Metallösungen," Annalen der Physik, vol. 330, no. 3, pp. 377-445, 1908. 

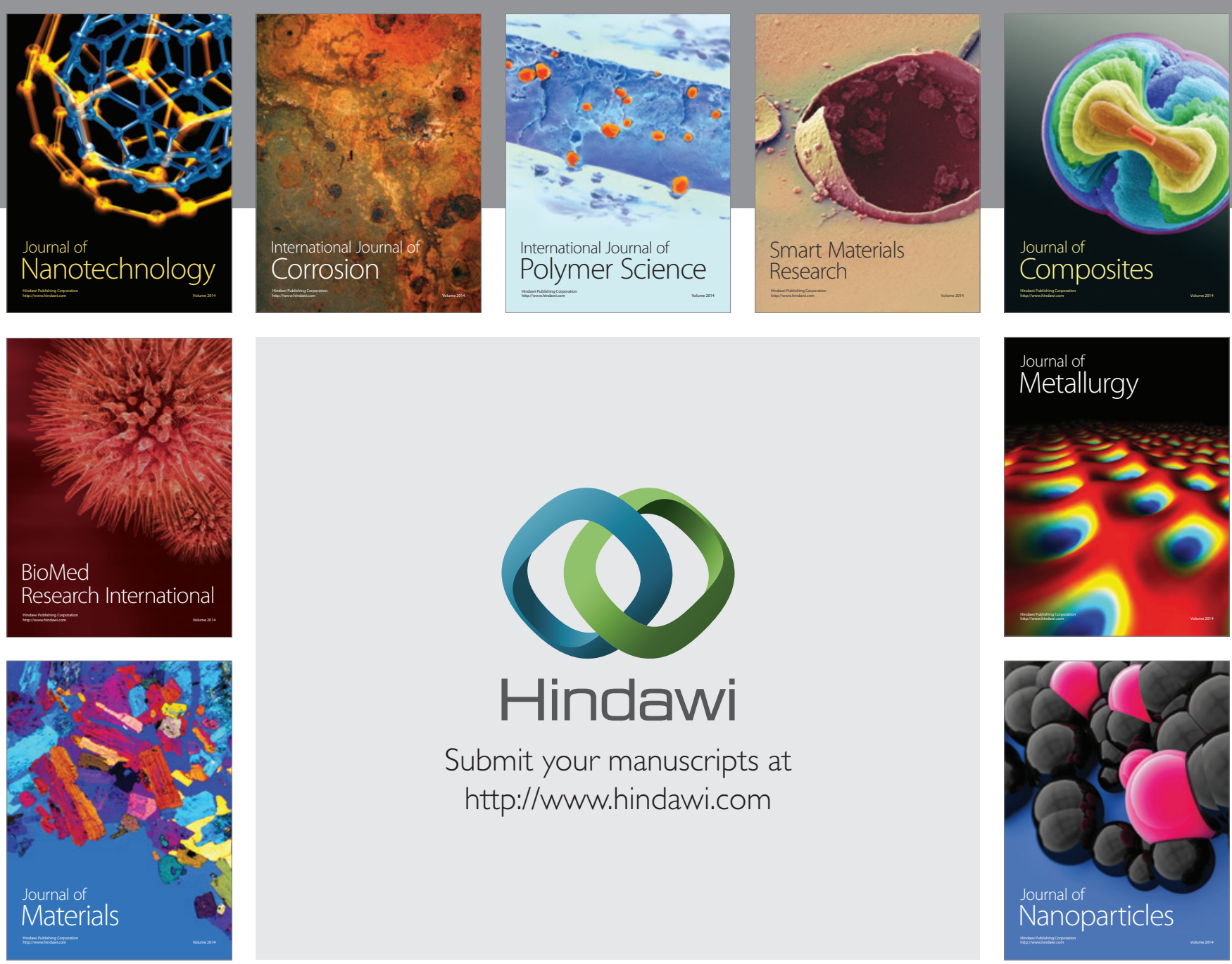

Submit your manuscripts at http://www.hindawi.com
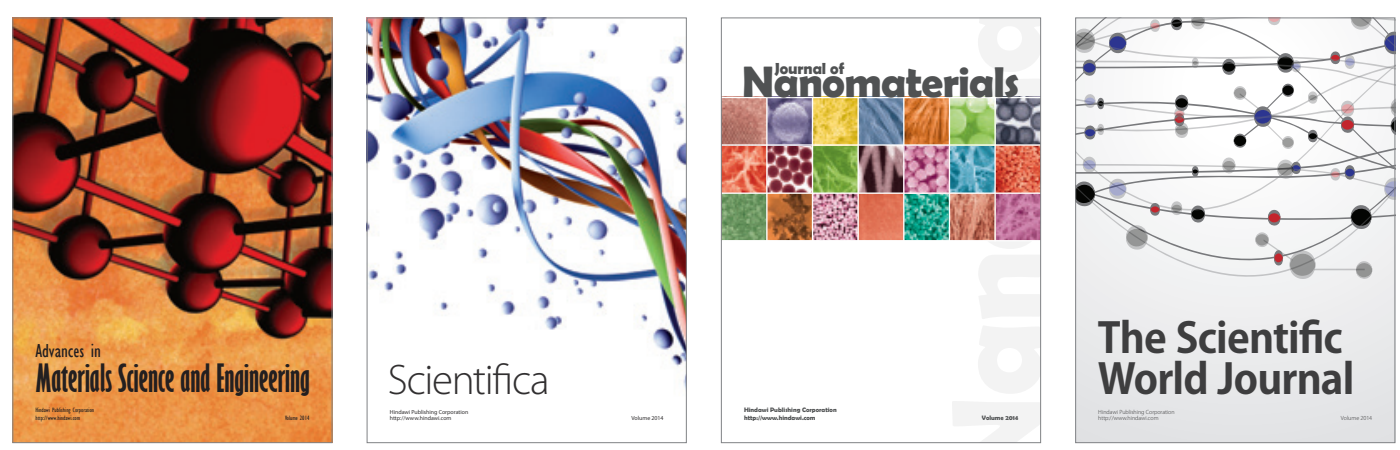

\section{The Scientific World Journal}
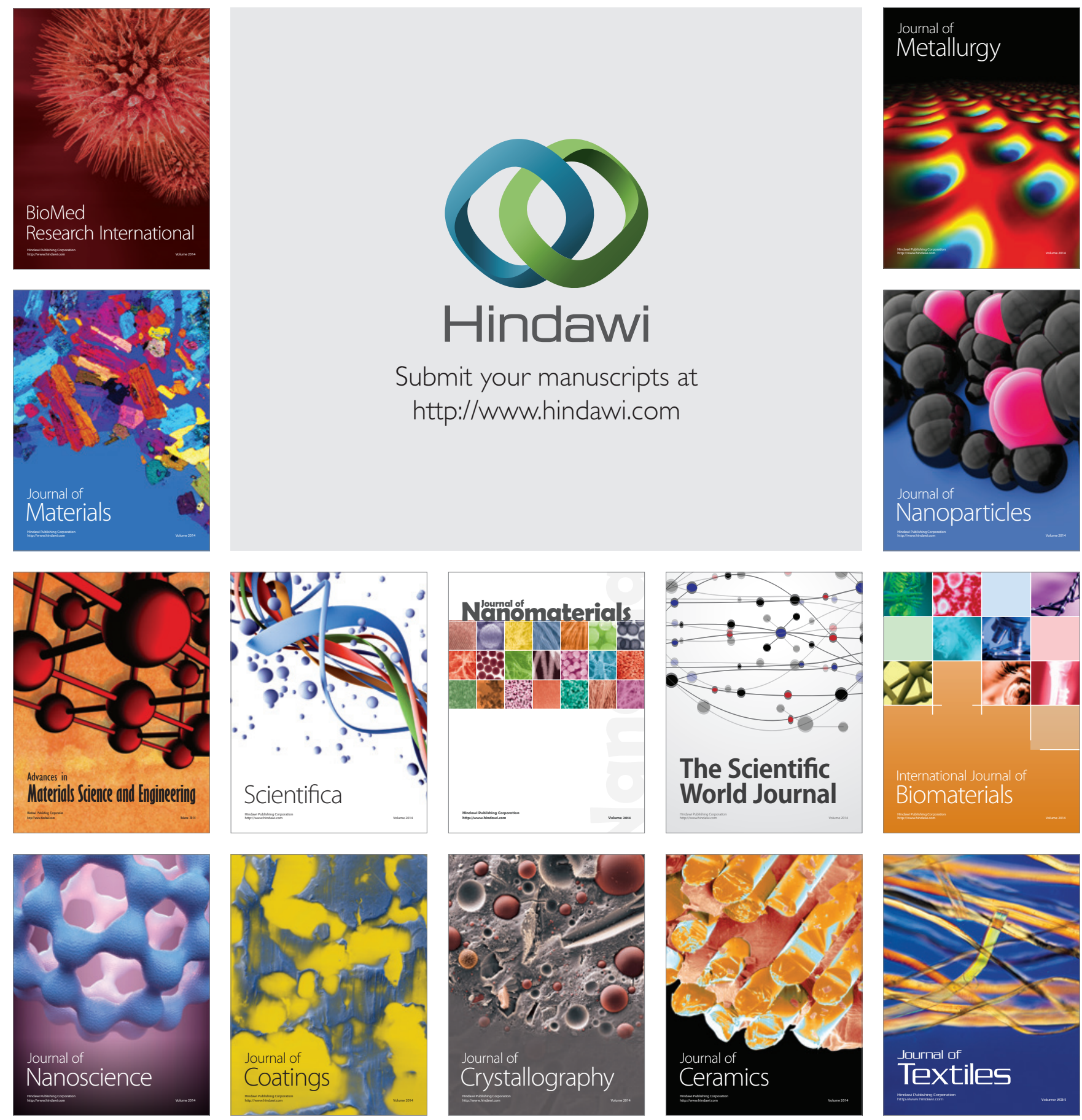